\title{
Decomposition of Dimethoate and Omethoate in Aqueous Solutions - Half-Life, Neurotoxicity and Mechanism of Hydrolysis
}

\author{
Vladan Anićijević1,2, Milena Petković ${ }^{2}$ Igor A. Pašti² and Tamara Lazarević-Pašti ${ }^{3, *}$ \\ 1 Military Technical Institute (VTI), RatkaResanovića 1, 11000 Belgrade, Serbia; \\ 2 University of Belgrade - Faculty of Physical Chemistry, Studentskitrg 12-16, 11158 Belgrade, Serbia \\ 3 Department of Physical Chemistry, "VINCA" Institute of Nuclear Sciences - National Institute of the Re- \\ public of Serbia, University of Belgrade, Mike Alasa 12-14, 11000 Belgrade, Serbia \\ * Correspondence: lazarevictlj@yahoo.com; tamara@vin.bg.ac.rs
}

\begin{abstract}
Simple Summary: Once the pesticides are released in the environment, a battle starts to remove them and prevent their negative effects on the species for which their use was not planned. For example, organophosphate pesticide dimethoate inhibits an important enzyme in the nervous system and in such a way affects all the organisms which have that enzyme. It includes humans, while its toxicity towards honey bees is extreme. To make the situation worse, if dimethoate is oxidized to omethoate, it becomes 100 times more toxic. However, if we understand how dimethoate transforms and decomposes in the environment, we can develop a strategy for protecting endangered organisms and plan the removal of dimethoate efficiently. Here we investigated how dimethoate decomposes in aqueous solutions and a model of contaminated water, depending on whether the solution is acidic or alkaline, in other words, its $\mathrm{pH}$. We found that dimethoate and omethoate decompose rapidly in alkaline solutions, but they are very stable in acidic ones and explained this difference using atomic-level modeling. Using the obtained data, now it is possible to predict how long dimethoate and omethoate will be present in contaminated water and how toxic this water will be depending on its $\mathrm{pH}$.
\end{abstract}

\begin{abstract}
Organophosphate pesticides are used in large quantities. However, they exhibit toxic effects on non-target organisms. Dimethoate and its oxo-analog omethoate inhibit acetylcholinesterase and are toxic for mammals. Moreover, they show extreme toxicity for bees. Once in the environment, they undergo chemical transformations and decomposition. We show that dimethoate and omethoate decompose rapidly in alkaline aqueous solutions (half-lives 5.7 and 0.89 days) but are highly stable in acidic solutions (half-lives 124 and 104 days). These differences are explained using quantum chemical calculations, indicating that a weaker P-S bond in omethoate is more susceptible to hydrolysis, particularly at a high $\mathrm{pH}$. The toxicity of these pesticides solutions decreases over time, indicating that no or very little highly toxic omethoate is formed during hydrolysis. Presented data can be used to predict dimethoate and omethoate concentrations in contaminated water depending on $\mathrm{pH}$. Presented results suggest that alkaline hydrolysis of organophosphates has an advantage over other techniques for their removal since there is no risk of omethoate accumulation and increased toxicity of contaminated water.
\end{abstract}

Keywords: organophosphate; pesticide; $\mathrm{pH}$ stability; toxicity; Apis mellifera;

\section{Introduction}

Dimethoate(Figure 1, a) is an organophosphorus pesticide (OP) with contact and systemic action. It is in use against many insects in agriculture and the housefly's control. Dimethoate is known for its moderate toxicity to mammals. The US EPA classified it as a possible human carcinogen based on tumor occurrence in exposed mice (US EPA, 
Interim Re-registration Eligibility Decision for Dimethoate. US EPA, Washington DC 20460 2006.). Like other organophosphates, the acute toxicity of dimethoate is caused by its inhibition of acetylcholinesterase (AChE) [1]. The oxidation of dimethoate leads to the formation of its oxo-analog, omethoate (Figure 1, b), which is more toxic towards acetylcholinesterase than the respective parent compound. Heavy contamination of aquatic ecosystems by dimethoate can lead to mass mortality of fish and other organisms. Omethoate may also be found in the environment due to different oxidizing agents in water and soil [1]].

The domesticated honey bees (Apis mellifera L.) are considered one of the most important pollinators for agricultural crops and wild plants worldwide. However, in Europe and North America, honey bee populations have been noticeable declining during the last few decades [2]. Besides parasites, pathogens, poor nutrition, queen failure, migratory stress, and habitat loss, the decline of pollinator species is related to pesticide use in agricultural landscapes []ㅡ. The sensitivity of domesticated honey bees to dimethoate is well-known for many years $[\underline{4}, \underline{5}]$. Furthermore, many recent studies found that dimethoate is highly toxic to bees [ 6 ]. The LD50 of dimethoate to honey bees is estimated to be $180 \mathrm{ng} / \mathrm{bee[}[\mathrm{7}]$. In concentrations as low as $0.2 \mu \mathrm{g} / \mathrm{g}$, dimethoate can decrease bee species weights []], induce larval mortality [ㅇ], and injure the hypopharyngeal glands of nurse bees $[\underline{9} \underline{10}]$. Besides, exposure to dimethoate has a negative impact on the structure and composition of the gut microbiota of honey bees [11]. Consequently, these changes induce alterations in bees' nutrition, metabolism, and behavior[12-14].

For all mentioned reasons, it is important to have a thorough understanding of the environmental fate of dimethoate and its analog omethoate to mitigate the negative impacts on the environment and the population of bees.

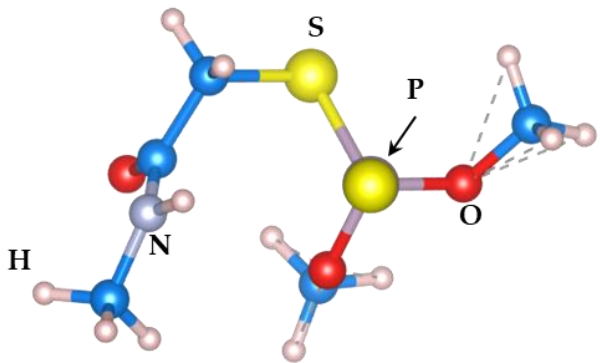

(a)

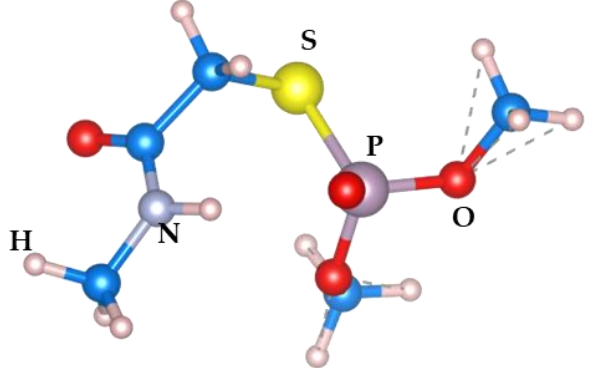

(b)

Figure 1. The structures of dimethoate (a) and omethoate (b) optimized using first-principles calculations (see further). The structures are presented using a ball and stick model. The following color code is used: blue - carbon, red - oxygen, purple - phosphorus, lite blue - nitrogen, white hydrogen, and yellow - sulfur.

Due to dimethoate's high water solubility and low soil persistence, its potential to runoff into surface waters and leaching into groundwater is high [15]. The most important degradation pathways of dimethoate in the environment are hydrolysis, photolysis and microbiological degradation [15]. The photocatalytic oxidation and microbial metabolism of dimethoate often have omethoate as the final product, which is not desirable due to its extreme toxicity. On the other hand, hydrolytic degradation is the main inactivating pathway of dimethoate in the environment and typically gives no omethoate as the final product. The hydrolysis of dimethoate is mostly dependent on the temperature and $\mathrm{pH}[\underline{16}]$.

Surprisingly, the systematic data for dimethoate and omethoate $\mathrm{pH}$-dependent stability are missing in the literature. Thus, the aim of the present work is to investigate the kinetics of dimethoate and omethoate hydrolysis in aqueous media at different $\mathrm{pH}$ (from 3 to 9) and explain the observed trends. In addition, the work thoroughly ad- 
dresses the toxicity of dimethoate and omethoate hydrolysis products, which is essential for tailoring efficient strategies for dimethoate and omethoate removal from water.

\section{Materials and Methods}

\subsection{Stability of dimethoate and omethoate in aqueous buffer solution}

The stability of dimethoate and omethoate on different $\mathrm{pH}$ was investigated in 50 $\mathrm{mM}$ phosphate buffer solution with $\mathrm{pH}$ ranging from 3 to 9 . The solutions of OPs were incubated at a temperature of $25{ }^{\circ} \mathrm{C}$ in a laboratory orbital shaker (Orbital Shaker-Incubator ES-20, Grant-bio) for 70 days. The concentrations of investigated OPs were measured as described below in aliquots taken at relevant time points.

\subsection{UPLC analysis}

For measuring the concentration of dimethoate and omethoate, Waters ACQUITY Ultra Performance Liquid Chromatography (UPLC) system, coupled with a tunable UV detector controlled by the Empower software, was used. Chromatographic separations were run on an ACQUITY UPLC ${ }^{\mathrm{TM}}$ BEH C18 column with the dimensions $1.7 \mu \mathrm{m}, 100$ $\mathrm{mm} \times 2.1 \mathrm{~mm}$ (Waters). The analyses of dimethoate and omethoate solutions were done under isocratic conditions with a mobile phase consisting of $10 \%$ acetonitrile and $90 \%$ water $(\mathrm{v} / \mathrm{v})$. The eluent flow rate was $0.25 \mathrm{~mL} \mathrm{~min}^{-1}$, and the injection volume was $5 \mu \mathrm{L}$. Optical detection for both OP was done at $200 \mathrm{~nm}$. Under described conditions, retention times of dimethoate and omethoate were $2.65 \pm 0.05 \mathrm{~min}$ and $1.12 \pm 0.05 \mathrm{~min}$, respectively.

\subsection{Toxicity measurements}

AChE inhibition measurements were performed to follow and quantify changes in the toxicity of dimethoate and omethoate and investigate if there are any transformations of OPs into more toxic forms upon hydrolysis on different $\mathrm{pH}$. These transformation products could exert harmful effects at concentrations below the detection limits of UPLC. AChE activity was assayed according to modified Ellman's procedure [17]. The method is described in detail in our previous work[18], and here we provide it for the sake of completeness. The in vitro experiments were performed by exposure of 0.5 IU commercially purified AChE from electric eel to OP solutions obtained in adsorption experiments at $37^{\circ} \mathrm{C}$ in $50 \mathrm{mM} \mathrm{PB} \mathrm{pH} 8.0$ (final volume $0.650 \mathrm{~mL}$ ). The enzymatic reaction was started by the addition of acetylcholine-iodide (AChI) in combination with 5,5'-dithio-bis(2-nitrobenzoic acid) (DTNB) as a chromogenic reagent and allowed to proceed for 8 min until stopped by 10\% sodium dodecyl sulfate (SDS). The enzymatic reaction product, thiocholine, reacts with DTNB and forms 5-thio-2-nitrobenzoate, whose optical adsorption was measured at $412 \mathrm{~nm}$. It should be noted that in these measurements, the enzyme concentration was constant and set to give an optimal spectrophotometric signal. Physiological effects were quantified as AChE inhibition given as:

$$
\text { AChE inhibition }=100 \times\left(A_{0}-A\right) / A_{0},
$$

where $A_{0}$ and $A$ stand for the AChE activity in the absence of OP and the one measured after the exposure to a given OP.

\subsection{Theoretical calculations}

All quantum chemical calculations were performed with the Gaussian software[19] at the M06-2X/6-311++G(d,p)[20-25] level using an ultrafine integration grid. Bader charges were obtained with the AIMAll[26] program package. The solvent (water) was added implicitly using the polarizable continuum model[27].

\section{Results}




\subsection{Dimethoate and omethoate decomposition over time}

The concentration of dimethoate and omethoate was monitored in tap water and phosphate buffers with $\mathrm{pH}$ ranging from 3 to 9, as described in Section 2.2. for70 days using UPLC analysis. PDA signals of dimethoate and omethoate at $\mathrm{pH} \mathrm{3,6}$ and 9 immediately after starting the experiments (Day $0,25^{\circ} \mathrm{C}$ ) arepresented in Figure 2.

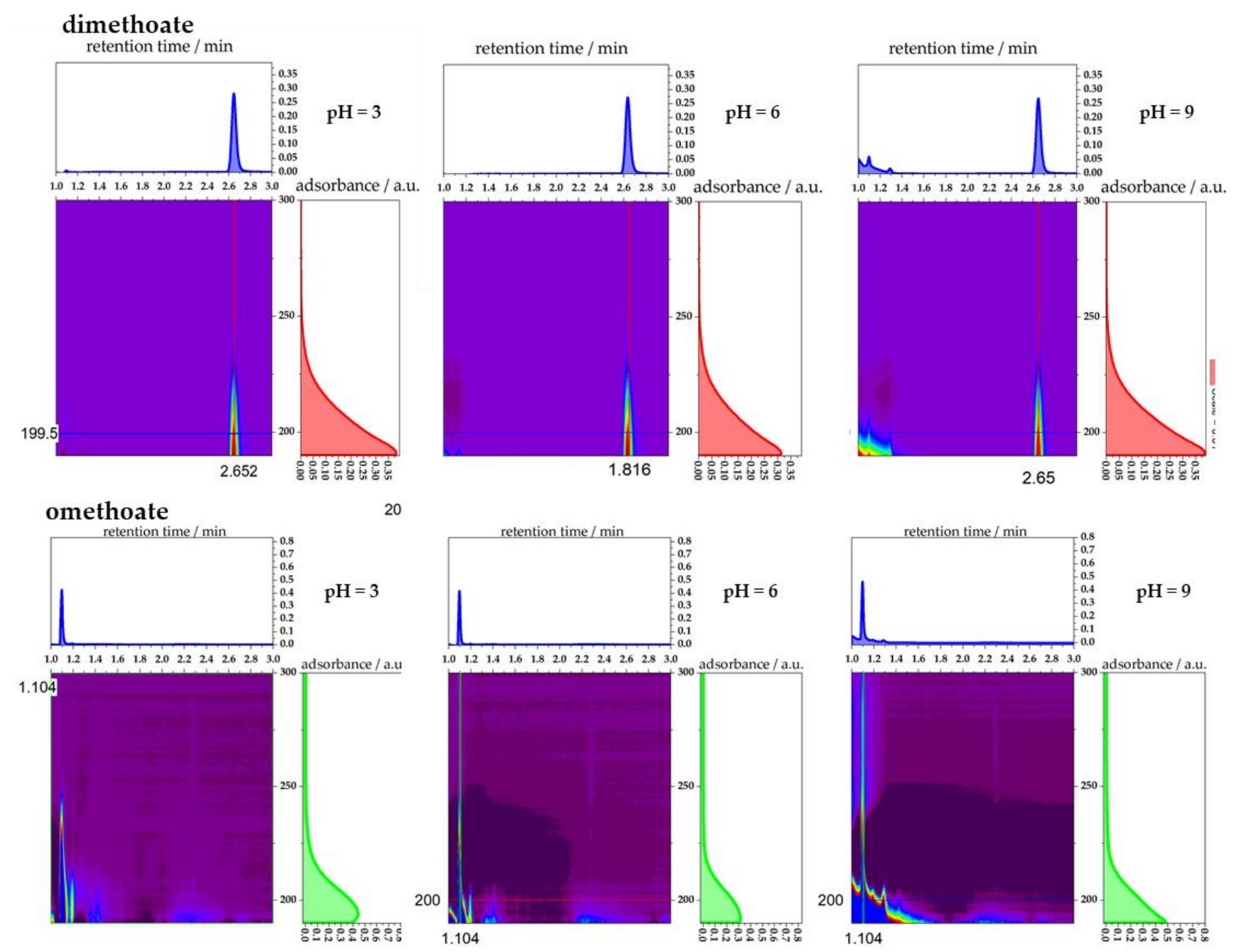

Figure 2. PDA signals of dimethoate and omethoate at three different $\mathrm{pH}$ values immediately after starting the experiments (Day 0, temperature $25^{\circ} \mathrm{C}$ ).

The time dependence of dimethoate and omethoate concentration is presented in Figure 3. It was shown that the spontaneous concentration decay of dimethoate (Figure 3 , a) and omethoate (Figure3,b) over time is rather fast in buffers with neutral and alkaline pHand tap water ( $\mathrm{pH}$ 6.5). On the other hand, in buffers with acidic $\mathrm{pH}$, the decrease of both OPs concentrations was also noticeable but at a lower rate. Different rate orders for the hydrolysis process were checked. In all the cases, the decay of OPs' concentration fitted the best to the exponential one and followed the pseudo-first-order kinetics. Hence, the hydrolysis rate constants $\left(k_{\mathrm{h}}\right)$ were obtained by direct fitting the experimental data into the equation:

$$
C_{\mathrm{t}}=C_{0} e^{-k_{\mathrm{h}} t}
$$

where $C_{t}$ and $C_{0}$ are the remaining OPs concentrations at a given time $(t)$ and the initial OPs concentration. The obtained rate constants are given in Table 1 . The presented data shows that hydrolysis rate constants are decreasing with the $\mathrm{pH}$ of OP solution in all studied cases. Also, for the same $\mathrm{pH}$ in neutral and alkaline conditions, the rate con- 
stants were higher for dimethoate than for omethoate by a factor of $4.5-6.5$. The hydrolysis rate constants were also higher for dimethoate in the acidic conditions, but the difference was not as prominent (up to a factor of 2).

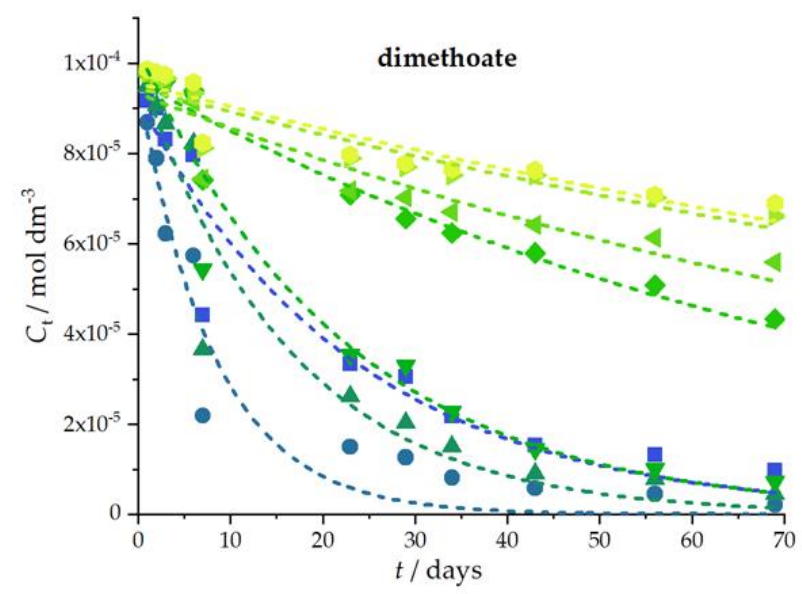

(a)

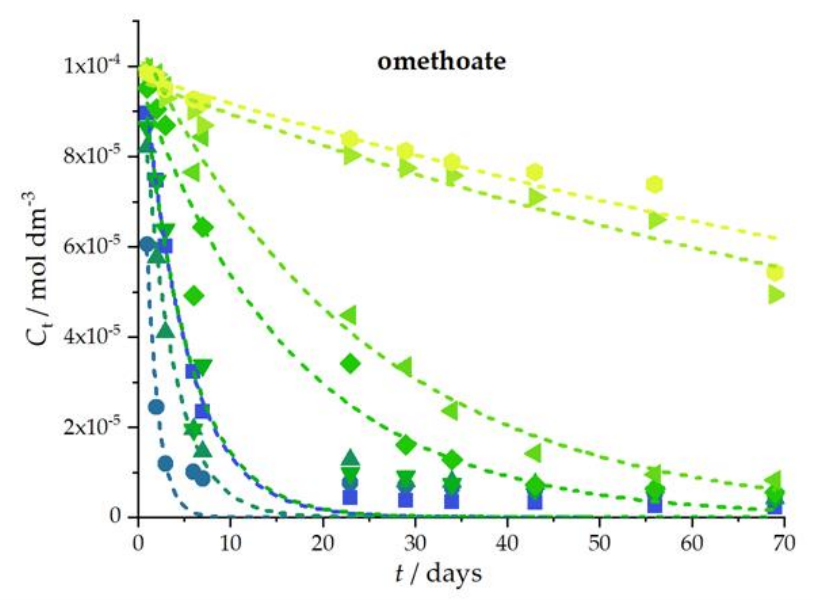

(b)

Figure 3.Dependence of dimethoate (a) and omethoate (b) concentration over 70 days at $\mathrm{pH}$ from 3 to $9\left(25^{\circ} \mathrm{C}\right)$.

Table 1. The rate constants $\left(k_{\mathrm{h}}\right)$ calculated for the decay of dimethoate and omethoate in $50 \mathrm{mM}$ phosphate buffers with pH ranging from 3 to 9 and tap water. $R^{2}$ is provided as a measure of the quality of the fit.

\begin{tabular}{cccc}
\hline OP & $\mathbf{p H}$ & $\boldsymbol{k}_{\mathbf{h}} / \mathbf{d}^{-1}$ & $\boldsymbol{R}^{\mathbf{2}}$ \\
\hline 9 & $(1.21 \pm 0.30) \times 10^{-1}$ & 0.91 \\
dimethoate & 8 & $(0.61 \pm 0.12) \times 10^{-1}$ & 0.92 \\
7 & $(0.444 \pm 0.058) \times 10^{-1}$ & 0.95 \\
& 6 & $(1.22 \pm 0.12) \times 10^{-2}$ & 0.92 \\
& 5 & $(8.5 \pm 1.2) \times 10^{-3}$ & 0.85 \\
& 4 & $(5.79 \pm 0.79) \times 10^{-3}$ & 0.85 \\
& 3 & $(5.59 \pm 0.81) \times 10^{-3}$ & 0.83 \\
\hline \multirow{5}{*}{ Spiked tap water $(\mathrm{pH}=6.5)$} & $(4.27 \pm 0.65) \times 10^{-2}$ & 0.92 \\
\hline 9 & $(7.9 \pm 1.9) \times 10^{-1}$ & 0.84 \\
& 8 & $(3.04 \pm 0.53) \times 10^{-1}$ & 0.93 \\
& 7 & $(2.01 \pm 0.36) \times 10^{-1}$ & 0.94 \\
& 6 & $(5.93 \pm 0.85) \times 10^{-2}$ & 0.95 \\
& 5 & $(4.11 \pm 0.21) \times 10^{-2}$ & 0.991 \\
& 4 & $(8.04 \pm 0.67) \times 10^{-3}$ & 0.94 \\
& 3 & $(6.66 \pm 0.63) \times 10^{-3}$ & 0.93 \\
\hline & Spiked tap water $(\mathrm{pH}=6.5)$ & $(2.10 \pm 0.13) \times 10^{-2}$ & 0.993 \\
\hline
\end{tabular}

The obtained hydrolysis rate constants for dimethoate and omethoate were further used to determine the half-life $\left(t_{1 / 2}\right)$ of these OPs under the given experimental conditions. The half-lives were estimated as: 


$$
t_{1 / 2}=\frac{\ln 2}{k_{\mathrm{h}}}
$$

The results are presented in Figure 4.

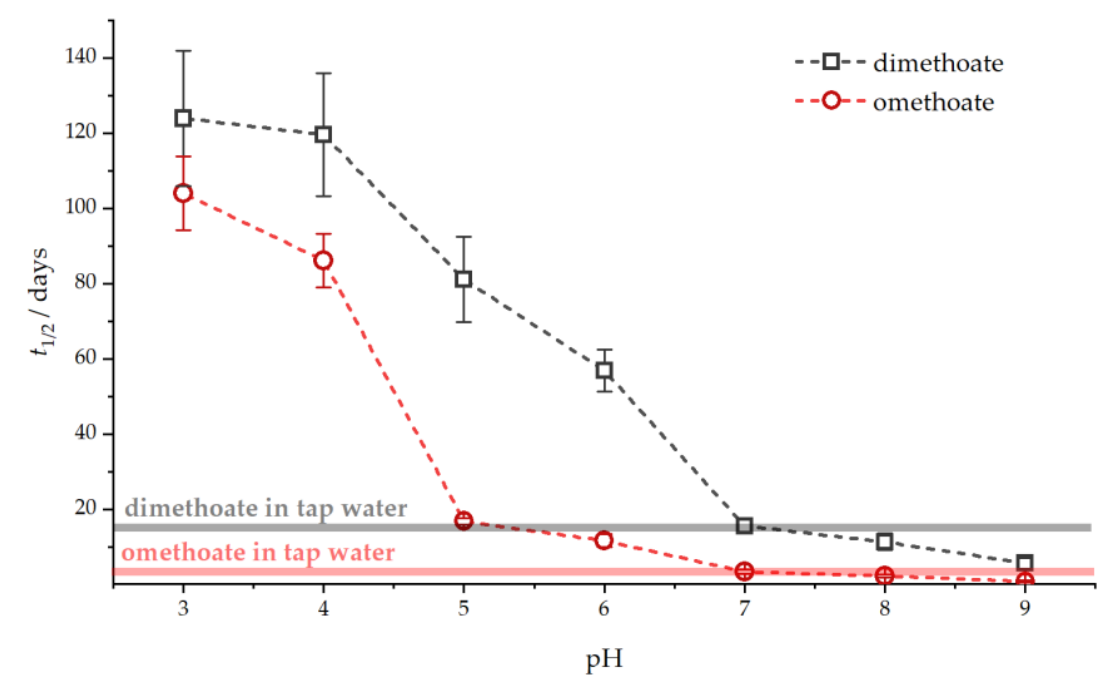

Figure 4.Estimated half-lives $\left(t_{1 / 2}\right)$ for dimethoate and omethoate in aqueous solutions as a function of $\mathrm{pH}\left(25^{\circ} \mathrm{C}\right)$. The rate constants for spiked tap water are indicated using horizontal lines.

\subsection{Toxicity of dimethoate and omethoate solutions over time}

Dimethoate solutions of initial concentration $1 \times 10^{-4} \mathrm{moldm}^{-3}$ were left in phosphate buffers ( $\mathrm{pH}$ ranging from 3 to $9,25^{\circ} \mathrm{C}$ ) and in tap water for ten days to monitor the toxicity of the spontaneous hydrolysis products. The toxicity of dimethoate solutions was estimated via the AChE inhibition test as described in Section 2.3. The aliquots for AChE inhibition testing were taken at the start and after 1, 2, 6 and 10 days. The results are given in Table 2.

Table 2. Toxicity of dimethoate solution over 10 days at $\mathrm{pH}$ from 3 to 9 . The initial concentration of dimethoate was $1 \times 10^{-4} \mathrm{~mol} \mathrm{dm}^{-3}\left(25^{\circ} \mathrm{C}\right)$

\begin{tabular}{cclccc}
\hline $\mathbf{p H}$ & \multicolumn{4}{l}{ AChE activity inhibition (\% of control) } \\
& Day 0 & Day 1 & Day 2 & Day 6 & Day 10 \\
\hline 3 & $34 \pm 2$ & $33 \pm 2$ & $33 \pm 2$ & $30 \pm 2$ & $31 \pm 2$ \\
4 & $36 \pm 3$ & $34 \pm 2$ & $34 \pm 1$ & $31 \pm 1$ & $30 \pm 2$ \\
5 & $34 \pm 2$ & $32 \pm 3$ & $30 \pm 2$ & $30 \pm 3$ & $30 \pm 1$ \\
7 & $32 \pm 1$ & $30 \pm 3$ & $30 \pm 2$ & $29 \pm 2$ & $29 \pm 1$ \\
8 & $32 \pm 2$ & $31 \pm 2$ & $29 \pm 2$ & $26 \pm 1$ & $25 \pm 2$ \\
9 & $32 \pm 2$ & $28 \pm 1$ & $24 \pm 1$ & $20 \pm 1$ & $17 \pm 1$ \\
Spiked tap water (pH 6.5) & $33 \pm 3$ & $22 \pm 2$ & $20 \pm 2$ & $13 \pm 1$ & $10 \pm 1$ \\
\end{tabular}

Besides the measurements of AChE activity inhibition for ten days, we have also mapped the AChE activity inhibition using the measured concentration of dimethoate over time $(\mathrm{Ct})$ and the inhibition curve of dimethoate. The results are presented in 
Figure 5, showing a rapid decrease of acute toxicity of water contaminated with dimethoate at $\mathrm{pH}$ above 7 .

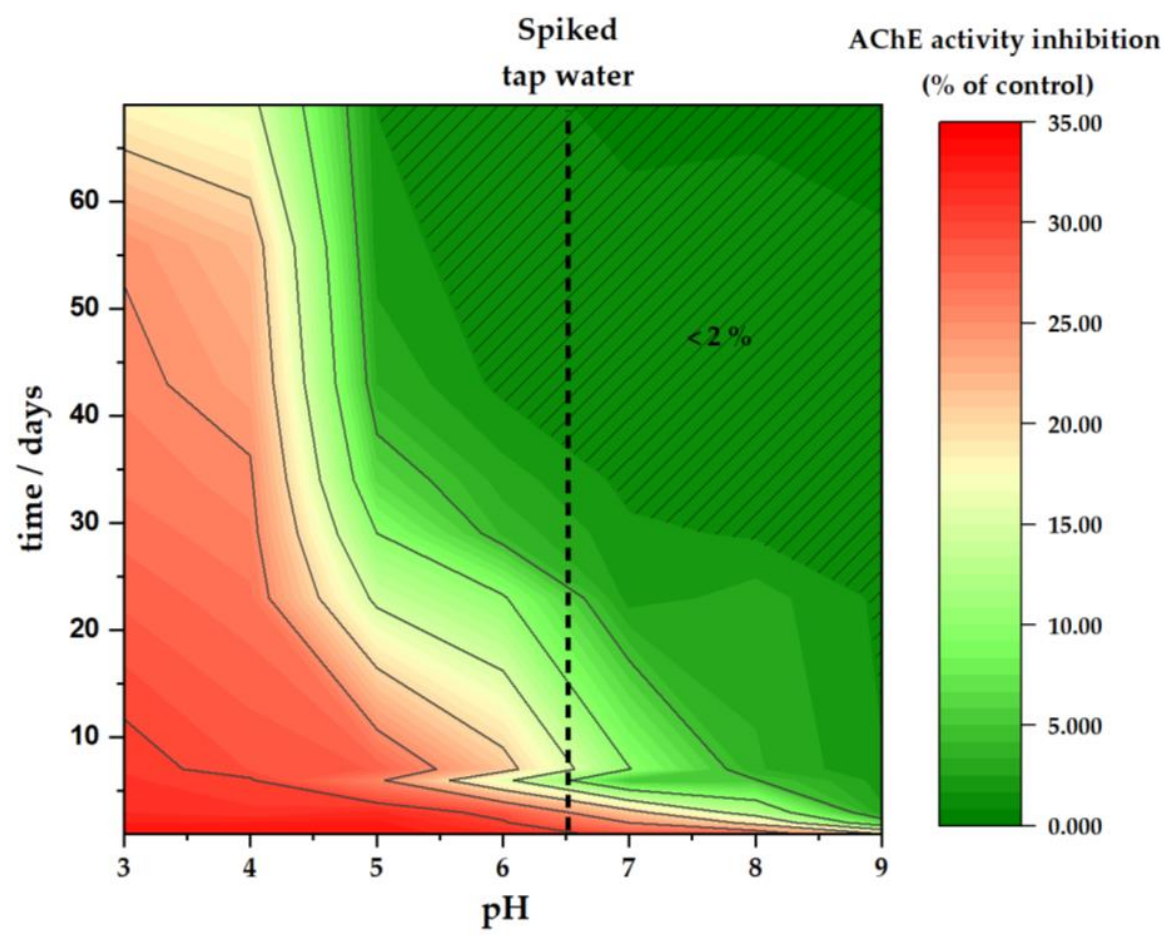

Figure 5. Maped AChE activity inhibition of water contaminated with dimethoate in the $\mathrm{pH}$ range 3-9 over 70 days. The patterned region shows the conditions under which AChE activity inhibition drops under $2 \%$ of control.

\subsection{Theoretical analysis of dimethoate hydrolysis in alkaline and acidic solutions}

First, we will address the alkaline hydrolysis of the two species. The reaction occurs according to the SN2 mechanism, leading to the P-S bond rupture. We performed partial optimizations for fixed O-P distances starting from $2.20 \AA$ (Figure 6). Electronic energy with respect to separated reactants and P-S bond length in partially optimized structures are presented in Table 3.System stabilization as the distance between the two reactants decreases is accompanied by P-S bond elongation.

Table 3.Results of partial optimization for fixed O-P bond distances. The values on the left/right correspond to dimethoate/omethoate. Electronic energy $\left(E_{\mathrm{el}}\right)$ is reported in $\mathrm{kcalmol}^{-1}$ with respect to separated reactants, while bond lengths $(r)$ are given in Å. "diss" stands for dissociation.

\begin{tabular}{ccc}
\hline$r(\mathrm{O}-\mathrm{P}) / \AA$ & $E_{\mathrm{el}} / \mathrm{kcalmol}^{-1}$ & $r(\mathrm{P}-\mathrm{S}) / \AA$ \\
\hline 2.20 & $-6.7 /-5.3$ & $2.232 / 2.230$ \\
2.10 & $-10.5 /-9.0$ & $2.255 / 2.258$ \\
2.00 & $-14.6 /-13.2$ & $2.281 / 2.291$ \\
1.90 & $-18.8 /-17.4$ & $2.311 / 2.334$ \\
1.80 & $-22.2 /-21.1$ & $2.356 / 2.403$ \\
1.70 & $-23.7 /$ diss & $2.425 /$ diss \\
1.60 & diss / - diss / -
\end{tabular}




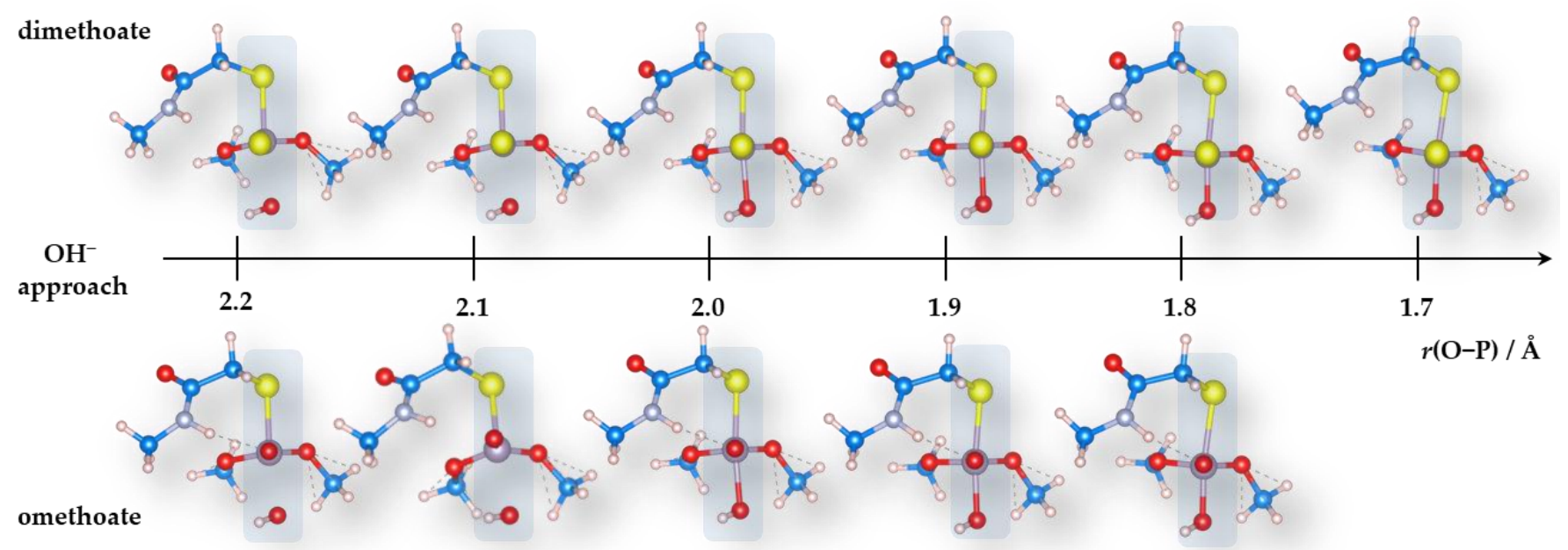

Figure 6. The attack of $\mathrm{OH}^{-}$ion approaching the $\mathrm{P}-\mathrm{S}$ moiety of dimethoate (top row) and O-P moiety omethoate (bottom row), leading to hydrolysis.

We find that in an acidic environment, the dissociation of the P-S bond takes place according to the following mechanism: (i)attachment of a single proton to the nitrogen atom, the carbonyl oxygen atom or the -S- atom results in the formation of a protonated species (in case $\mathrm{P}-\mathrm{S}\left(\mathrm{H}^{+}\right)-\mathrm{C}$ is formed, the P-S bond weakens, which is reflected in its lengthening by $0.221 \AA$ ); (ii) attaching another $\mathrm{H}^{+}$ion to the $-\mathrm{S}$ - atom breaks the P-S bond.

\section{Discussion}

The fundamental understanding of OP hydrolysis mechanisms is of uttermost importance for precise planning of OPs disposal and removal[27]. One of the main results of our work is that omethoate hydrolysis is faster compared to dimethoate. Using the results of theoretical calculations, this can be explained by the fact that starting from $r(\mathrm{O}-\mathrm{P})=2.10 \AA$, the $\mathrm{P}-\mathrm{S}$ bond is longer (i.e., weaker) in omethoate (Table 3). Moreover, at $r(\mathrm{O}-\mathrm{P})=1.70 \AA$, omethoate decomposes, whereas dimethoate still exists, although with a significantly weakened P-S bond (Figure 6). These results suggest that omethoate is less stable than dimethoate in the presence of hydroxide ions. Also, we find that hydrolysis is faster in alkaline media. We note that there are six electronegative atoms in dimethoate: three oxygen atoms, one nitrogen atom and two sulfur atoms. Charges of the $\mathrm{O}$ and $\mathrm{N}$ atoms are lower than $-1.000 e$, whereas charges of the $\mathrm{S}=$ and $-\mathrm{S}-$ atoms amount to -0.733 and $-0.251 e$, respectively. Hence, the reason for slow hydrolysis of dimethoate under acidic conditions lies in the presence of six electronegative atoms, while protonation of only specific sites (see section 3.3) leads to the P-S bond dissociation.

The results presented in Figure 4 show that the estimated half-lives for dimethoate and omethoate in an aqueous solution decrease with the increase of $\mathrm{pH}$ but not in a linear fashion. Above $\mathrm{pH}$ 6, the half-life is highly sensitive to the change of $\mathrm{pH}$. At $\mathrm{pH} 9$, half-lives of both dimethoate and omethoate are very small and rather close (5.7 days for dimethoate and 0.89 days for omethoate). As $\mathrm{pH}$ decreases, the difference between half-lives for studied OPs increases and reaches its maximum at $\mathrm{pH} 5$ (17 days for omethoate and 81 days for dimethoate). With $\mathrm{pH}$ further changing to more acidic, this difference decreases. However, the half-lives for dimethoate are still appreciably higher than for omethoate (104 days for omethoate and 124 days for dimethoate at $\mathrm{pH} 3$ ). From the theoretical calculations, it is obvious that the structure of OP determines its stability under different $\mathrm{pH}$ conditions. Dimethoate is more stable than omethoate, which is similar to the cases of parathion and paraoxon and diazinon and diazoxon[28,29]. Parathion and diazinon (and their oxo-forms) have certain similarities, both containing 
aromatic rings bonded to the $\mathrm{SPO}_{3}$ moiety. However, dimethoate has aliphatic groups bonded to $\mathrm{S}_{2} \mathrm{PO}_{2}$ moiety. Also, the stability of these molecules differs in terms of $\mathrm{pH}$ dependence. For parathion and paraoxon, the half-lives decrease monotonically with $\mathrm{pH}$, but diazinon and diazoxon reach maximum in near-neutral solutions. Obviously, careful work is needed to fully resolve the link between the OPs structure and their stability under different $\mathrm{pH}$ in aqueous media.

Importantly, it is obvious that matrix effects in spiked tap water have a minor impact on the rate of hydrolysis (Table 2). Thus, the presented results can be used to reliably estimate dimethoate and omethoate half-lives in aqueous media based on $\mathrm{pH}$ only.

Finally, from the data presented in Table 2, it can be concluded that the inhibition of AChE activity decreased monotonically over time in all samples tested. While in some cases, small amounts of omethoate can be found at the beginning of the experiment (see Figure 1, PDA signals for dimethoate at $\mathrm{pH}$ 9), there is no accumulation of omethoate which would cause an increase of toxicity of contaminated aqueous solutions. As there is no accumulation of omethoate, although we have not determined the hydrolysis products, it can be safely concluded that omethoate is not the hydrolysis product or that it is being produced in minor quantities. Namely, one should keep in mind that it is roughly 100 times [1] more toxic than dimethoate and that only 1 percent dimethoate converted to omethoate would result in doubled toxicity. Therefore, by combining the results of $\mathrm{pH}$ stability of dimethoate with its AChE inhibition curve, AChE activity inhibition of contaminated water samples can be reliable predicted for the period of up to 70 days, as done in Figure 5.

\section{Conclusions}

Dimethoate and omethoate hydrolyze at different rates, and the hydrolysis of omethoate is faster than hydrolysis of dimethoate. During hydrolysis, there is no accumulation of omethoate, which is much more toxic than dimethoate. As the rate constant of dimethoate and omethoate rapidly increases with $\mathrm{pH}$ in alkaline media, it is suggested that alkaline hydrolysis is a suitable way for the removal of dimethoate from water, as, unlike, for example, some cases of microbial degradation and photocatalytic oxidation, it does not lead to accumulation of more toxic product during the degradation process. Hence, if alkaline hydrolysis is used for dimethoate (and omethoate) removal, no special care should be taken for monitoring the degradation process as the risk for the formation of toxic products is nonexistent. The matrix effects in tap water have a negligible impact on dimethoate and omethoate hydrolysis rate, so the presented data can be safely used to estimate dimethoate and omethoate half-lives in contaminated water. Moreover, the toxicity data can be used to evaluate acute toxicity upon water contamination, measured as the AChE inhibition. The estimations for longer periods (beyond ten days) can be done using the combination of the stability data and the AChE inhibition curve for dimethoate.

Author Contributions: Conceptualization, T.L.P. and I.A.P.; formal analysis, V.A., M.P. and T.L.P.; resources, M.P. and I.A.P..; writing-original draft preparation, all authors; writing-review and editing, I.P., M.P. and T.L.P.; All authors have read and agreed to the published version of the manuscript.

Funding: T.L.P. acknowledges the support provided by the Serbian Ministry of Education, Science and Technological Development (Contract number: 451-03-9/2021-14/200017). V.A, M.P. and I.AP.acknowledge the support provided by the Serbian Ministry of Education, Science and Technological Development (Contract number: 451-03-68/2020-14/200146).

Conflicts of Interest: The authors declare no conflict of interest. 


\section{References}

1. Lazarević-Pašti, T.D.; Pašti, I.A.; Jokić, B.; Babić, B.M.; Vasić, V.M. Heteroatom-doped mesoporous carbons as efficient adsorbents for removal of dimethoate and omethoate from water. RSC Advances 2016, 6, 62128-62139, doi:10.1039/c6ra06736k.

2. Dai, P.; Jack, C.J.; Mortensen, A.N.; Bustamante, T.A.; Bloomquist, J.R.; Ellis, J.D. Chronic toxicity of clothianidin, imidacloprid, chlorpyrifos, and dimethoate to Apis mellifera L. larvae reared in vitro. 2019, 75, 29-36, doi:https://doi.org/10.1002/ps.5124.

3. Uhl, P.; Franke, L.A.; Rehberg, C.; Wollmann, C.; Stahlschmidt, P.; Jeker, L.; Brühl, C.A. Interspecific sensitivity of bees towards dimethoate and implications for environmental risk assessment. Scientific Reports 2016, 6, 34439, doi:10.1038/srep34439.

4. Waller, G.D.; Erickson, B.J.; Harvey, J.; Martin, J.H. Effects of Dimethoate on Honey Bees (Hymenoptera: Apidae) When Applied to Flowering Lemons. Journal of Economic Entomology 1984, 77, 70-74, doi:10.1093/jee/77.1.70 \%J Journal of Economic Entomology.

5. Waller, G.D.; Barker, R.J. Effects of Dimethoate on Honey Bee Colonies12. Journal of Economic Entomology 1979, 72, 549-551, doi:10.1093/jee/72.4.549 \%J Journal of Economic Entomology.

6. Decourtye, A.; Devillers, J.; Genecque, E.; Menach, K.L.; Budzinski, H.; Cluzeau, S.; Pham-Delègue, M.H. Comparative Sublethal Toxicity of Nine Pesticides on Olfactory Learning Performances of the Honeybee Apis mellifera. Archives of Environmental Contamination and Toxicology 2005, 48, 242-250, doi:10.1007/s00244-003-0262-7.

7. Ghini, S.; Fernández, M.; Picó, Y.; Marín, R.; Fini, F.; Mañes, J.; Girotti, S. Occurrence and Distribution of Pesticides in the Province of Bologna, Italy, Using Honeybees as Bioindicators. Archives of Environmental Contamination and Toxicology 2004, 47, 479-488, doi:10.1007/s00244-003-3219-y.

8. López, J.H.; Krainer, S.; Engert, A.; Schuehly, W.; Riessberger-Gallé, U.; Crailsheim, K. Sublethal pesticide doses negatively affect survival and the cellular responses in American foulbrood-infected honeybee larvae. Scientific Reports 2017, 7, 40853, doi:10.1038/srep40853.

9. Lensing, W. Changes in honey bee workers after feeding them with sublethal doses of dimethoate. Apidologie (France) 1986.

10. Aupinel, P.; Fortini, D.; Michaud, B.; Marolleau, F.; Tasei, J.N.; Odoux, J.F. Toxicity of dimethoate and fenoxycarb to honey bee brood (Apis mellifera), using a new in vitro standardized feeding method. Pest Management Science: formerly Pesticide Science 2007, 63, 1090-1094.

11. Kakumanu, M.L.; Reeves, A.M.; Anderson, T.D.; Rodrigues, R.R.; Williams, M.A. Honey bee gut microbiome is altered by in-hive pesticide exposures. Frontiers in microbiology 2016, 7, 1255.

12. Zheng, H.; Powell, J.E.; Steele, M.I.; Dietrich, C.; Moran, N.A. Honeybee gut microbiota promotes host weight gain via bacterial metabolism and hormonal signaling. Proceedings of the National Academy of Sciences 2017, 114, 4775-4780.

13. Jones, J.C.; Fruciano, C.; Marchant, J.; Hildebrand, F.; Forslund, S.; Bork, P.; Engel, P.; Hughes, W.O.H. The gut microbiome is associated with behavioural task in honey bees. Insectes Sociaux 2018, 65, 419-429, doi:10.1007/s00040-018-0624-9.

14. Yang, Y.; Ma, S.; Yan, Z.; Liu, F.; Diao, Q.; Dai, P. Effects of three common pesticides on survival, food consumption and midgut bacterial communities of adult workers Apis cerana and Apis mellifera. Environmental Pollution 2019, 249, 860-867.

15. Van Scoy, A.; Pennell, A.; Zhang, X. Environmental fate and toxicology of dimethoate. Reviews of Environmental Contamination and Toxicology Volume 237 2016, 53-70.

16. $\quad$ https://www.who.int/water sanitation health/WHS WWD2010 guidelines 20106 en.pdf?ua=1. 
17. Ellman, G.L.; Courtney, K.D.; Andres Jr, V.; Featherstone, R.M. A new and rapid colorimetric determination of acetylcholinesterase activity. Biochemical pharmacology 1961, 7, 88-95.

18. Lazarević-Pašti, T.; Čolović, M.; Savić, J.; Momić, T.; Vasić, V. Oxidation of diazinon and malathion by myeloperoxidase. Pesticide biochemistry and physiology 2011, 100, 140-144.

19. Frisch, M.; Trucks, G.; Schlegel, H.B.; Scuseria, G.E.; Robb, M.A.; Cheeseman, J.R.; Scalmani, G.; Barone, V.; Mennucci, B.; Petersson, G. gaussian 09, Revision d. 01, Gaussian. Inc., Wallingford CT2009, 201.

20. Zhao, Y.; Truhlar, D.G. The M06 suite of density functionals for main group thermochemistry, thermochemical kinetics, noncovalent interactions, excited states, and transition elements: two new functionals and systematic testing of four M06-class functionals and 12 other functionals. Theoretical chemistry accounts 2008, 120, 215-241.

21. McLean, A.D.; Chandler, G.S. Contracted Gaussian basis sets for molecular calculations. I. Second row atoms, Z=11-18. The Journal of chemical physics 1980, 72, 5639-5648.

22. Krishnan, R.; Binkley, J.S.; Seeger, R.; Pople, J.A. Self-consistent molecular orbital methods. XX. A basis set for correlated wave functions. The Journal of chemical physics 1980, 72, 650-654.

23. Clark, T.; Chandrasekhar, J.; Spitznagel, G.W.; Schleyer, P.V.R. Efficient diffuse function-augmented basis sets for anion calculations. III. The 3-21+ G basis set for first-row elements, Li-F. Journal of Computational Chemistry 1983, 4, $294-301$.

24. Frisch, M.J.; Pople, J.A.; Binkley, J.S. Self-consistent molecular orbital methods 25. Supplementary functions for Gaussian basis sets. The Journal of chemical physics 1984, 80, 3265-3269.

25. Blaudeau, J.-P.; McGrath, M.P.; Curtiss, L.A.; Radom, L. Extension of Gaussian-2 (G2) theory to molecules containing third-row atoms K and Ca. The Journal of chemical physics 1997, 107, 5016-5021.

26. Keith, T.A. AIMAll (Version 19.02. 13). TK Gristmill Software, Overland Park KS, USA 2019, 2019.

27. Tomasi, J.; Mennucci, B.; Cammi, R. Quantum mechanical continuum solvation models. Chemical reviews 2005, 105, 2999-3094.

28. Lockridge, O.; Verdier, L.; Schopfer, L.M. Half-life of chlorpyrifos oxon and other organophosphorus esters in aqueous solution. Chemico-biological interactions 2019, 311, 108788.

29. Faust, S.D.; Gomaa, H.M. Chemical hydrolysis of some organic phosphorus and carbamate pesticides in aquatic environments. Environmental letters 1972, 3, 171-201. 